\title{
Surgical Implant Efficiency
}

\author{
Brenda Beck
}

\section{Key Concepts}

- Operating room efficiency may save time and money without compromising quality.

- "Lean production" or removing steps that are of little value to the patient can decrease waiting time for patients and improve quality of care.

- Standardized operating room layouts as well as equipment can decrease the likelihood of slowing the procedure or the potential of harming the patient.

- Basic knowledge of operating room surgical equipment, nomenclature, and technique are imperative for the operating physician to master.

\section{Introduction}

Due to rising health-care costs, maximizing perioperative efficiency is paramount to reduce waste, decrease personnel cost, and increase financial performance. A lean process strategy (as reflected by the Toyota Production System) eliminates "waste" that may absorb time, personnel, or resources that do not add to the value or efficiency of patient care. On-time starts, eliminating the collection of redundant patient information before the start of a procedure, and reducing operating room turnover times may improve overall efficiency without compromising patient quality of care.
Standardized operating room layouts and procedural as well as surgical equipment may help to maintain workflow in the operating room, decrease patient operating room exposure time (which can lead to a lower rate and risk of infection to the patient), and decrease the potential for operating room personnel mistakes or potential errors that may harm the patient.

Having a standard surgical pull sheet for an implant procedure may be helpful in preventing delays and longer surgical exposure time for the patient and can help to maximize efficiency and quality of care.

The physician should have a basic knowledge of the nomenclature of surgical equipment and the basic tools used during a surgical implant procedure (see Appendix 3). A basic minor surgery set should include, but not limited to, instruments designed to cut, grasp, control bleeding, retract, and clamp. Wound care supplies should also be readily available. Different wound dressing techniques can be at the discretion of the physician.

\section{Conclusion}

Creating a responsive surgical care delivery is helpful on many fronts; most importantly, it optimizes patient care by reducing surgical site infection. The patient centric strategy associated with the tasking of the personnel during the surgery will reduce costs of the surgery site and improve patient care and physician efficiency.

\section{Suggested Reading}

1. Arakelian E, Gunningberg L, Larsson J. How operating room efficiency is understood in a surgical team: a qualitative study. Int J Qual Health Care. 2011;23(1):100-6.

2. Cima RR, Brown MJ, et al. Use of lean and six sigma methodology to improve operating room efficiency in a high-volume tertiary-care academic medical center. Am Coll Surg. 2011;213(1):83-92.
University Hospitals, Department of Anesthesiology, Division of Pain Management, 11100 Euclid Ave, Cleveland, OH 44106, USA e-mail: Brenda.beck@uhhospitals.org 